\title{
Effect of taste context and ambient context changes on successive negative contrast
}

\author{
CHARLES F. FLAHERTY, KEVIN HRABINSKI, and PATRICIA S. GRIGSON \\ Rutgers University, New Brunswick, New Jersey
}

\begin{abstract}
Rats shifted from $32 \%$ sucrose to $4 \%$ sucrose lick less than rats that experience only the $4 \%$ solution. Previous experiments have found this negative contrast effect to be reduced ("disinhibited") by the addition of a novel tone in the postshift period. In Experiment 1 of this paper, the negative contrast effect was enhanced when a novel flavor was added to the sucrose solution in the postshift period. In Experiments 2-4, changes in the ambient context, even changes sufficient to produce disruptions in licking, did not alter the degree of negative contrast. These results suggest that (1) rats compare rewards across substantially different contexts, (2) contrast may serve to enhance taste neophobia, and (3) a disinhibitory effect may be confined to the presentation of punctate, nontaste, novel stimuli within a familiar context.
\end{abstract}

Rats shifted from a $32 \%$ to $4 \%$ sucrose solution consume less of the $4 \%$ solution than do animals that experience only the latter solution. Such "negative contrast" effects are pervasive in consummatory behavior, but the extent to which negative contrast is confined to the context in which different rewards are received has been little investigated. One study, which was concerned with the role of inhibition in contrast, showed that the introduction of a novel stimulus (a loud tone) concomitant with the shift led to a reduced contrast effect, but only on the second and subsequent postshift days (Lombardi \& Flaherty, 1978).

Other studies, using different contrast procedures in which animals regularly experienced two different reinforcers, showed that changes in the environment in which the animals received the reinforcers had relatively little effect on contrast (Flaherty \& Avdzej, 1976; Premack, 1969). Similarly, recent studies in traditional learning paradigms have indicated that changes in conditioning context have relatively little influence on responding to a previously reinforced signal, although responding to a nonreinforced signal may be substantially impaired by context shifts (Bouton, 1986, in press; Kaye, Preston, Szabo, Druiff, \& Mackintosh, 1987; Lovibond, Preston, \& Mackintosh, 1984).

The consummatory contrast procedure is different from these traditional conditioning studies in that there is no explicit signal for the sucrose solutions other than the context itself. Since the animals presumably must compare the postshift reward with the memory of the preshift reward in order to show a contrast effect (e.g., see Flaherty \& Lombardi, 1977; Gonzalez, Fernhoff, \& David, 1973;

This research was supported by NIMH Grant $\mathrm{MH}-40489$ and by a Charles and Johanna Busch Memorial grant. Comments made by the reviewers substantially enhanced the paper. Correspondence may be addressed to Charles Flaherty, Psychology Department, Busch Campus, Rutgers University, New Brunswick, NJ 08903.
Spear, 1967), the degree to which this memory must be associated with the preshift context is of some interest. The literature mentioned above suggests that rewards are compared across substantially different contexts when the animals repeatedly experience different rewards, but the role of context when the animals experience a single shift is not clear. The one "disinhibition" study suggests that introduction of a punctate stimulus change within a familiar context may diminish contrast, but the role of global changes in context has not been investigated.

In the present paper, we describe four experiments in which the postshift context was changed either by adding a novel taste to the postshift solution (Experiment 1) or by changing the ambient context to a moderate degree (Experiment 2), substantially (Experiment 3 ), or substantially with prior habituation to the new context (Experiment 4).

\section{EXPERIMENT 1}

An issue in consummatory negative contrast is the extent to which the decrement in licking following the shift from $32 \%$ to $4 \%$ sucrose represents a generalization decrement process-that is, a process related to the experience of a novel flavor and not to the loss of reward per se. It is unlikely that generalization decrement contributes a major portion of the variance in the consummatory contrast situation, since animals repeatedly shifted between $32 \%$ and $4 \%$ sucrose will continue to show negative contrast, apparently indefinitely, under a variety of shift conditions (Flaherty, Becker, \& Checke, 1983). However, flavor novelty may play some role, because animals exposed to a variety of differently flavored $32 \%$ sucrose solutions during the preshift period show a smaller negative contrast when shifted to an unflavored $4 \%$ solution than do animals without prior varied taste experience (Meinrath \& Flaherty, 1988).

There is another way in which contrast and flavor novelty may interact. Lombardi (1980) showed that 
animals exposed to a decrease in unflavored sucrose concentration were apparently sensitized to flavor novelty, showing greater decrements in licking when exposed to novel flavors than animals without prior contrast experience.

In the present experiment, we were concerned with the effect of adding a novel flavor to the postshift solution in animals without prior experience with varied flavors. At least two outcomes of such an experiment seemed possible. First, the novel flavor could serve as a context change or a disinhibitory stimulus, thus decreasing the size of contrast (cf. Lombardi \& Flaherty, 1978). Alternatively, the concomitant experience of reward shift plus novel flavor could serve to enhance contrast, as the Lombardi (1980) results might suggest.

\section{Method}

Subjects. Twenty-four male Sprague-Dawley-derived rats were used, 12 of which had previous experience in an insulin conditioning experiment. The animals were housed singly in hanging metal cages and were maintained on a 14:10-h light:dark cycle. The rats were deprived to $82 \%$ of their free-feeding weights for the duration of the experiment.

Apparatus. Testing was conducted in six identical metal grid cages $(24.5 \times 17.5 \times 18 \mathrm{~cm})$. A centrally located hole $(1 \mathrm{~cm}$ in diameter $) 7 \mathrm{~cm}$ above the floor was present in one wall of each cage. A graduated cylinder was placed outside of each chamber so that the orifice of the drinking spout was centered in the hole and flush with one wall of the chamber. Licking was recorded through a contact-relay circuit by microprocessors. White noise was continuously present in the room containing the apparatus.

Procedure. The experiment was conducted as a $2 \times 2$ factorial, varying shift condition (shifted or unshifted) and flavor condition (novel flavor or no novel flavor in the postshift period). The unshifted animals received $4 \%$ sucrose throughout the experiment, whereas the shifted animals received access to a $32 \%$ sucrose solution for 10 days and then a $4 \%$ sucrose solution for an additional 4 days. Daily sessions consisted of a 5-min period of access to the solutions, beginning with each animal's first lick

During the postshift period, half of the shifted and half of the unshifted animals had a novel flavor (Durkee's cherry flavor mixed at a $1: 50$ ratio) added to the $4 \%$ sucrose solution. (A pilot study had indicated that a 1:40 ratio produced a substantial lick decrement in both shifted and unshifted animals.)

\section{Results}

Terminal preshift (Day 10) and daily postshift lick frequencies are presented in the top panel of Figure 1, and the same data are expressed as a proportion of preshift lick frequency in the bottom panel of Figure 1. Because of the terminal preshift difference in lick frequency (when no flavors were present) between the flavor and no-flavor groups (top panel of Figure 1), the data were analyzed in terms of proportion of preshift lick frequencies. This analysis showed that there was an overall contrast effect $[32-4<4-4, F(1,15)=126.35, p<.001]$, an overall effect of the added flavor [flavor $<$ no flavor, $F(1,15)$ $=8.73, p<.001]$, and a reliable shift $\times$ flavor interaction $[F(1,15)=15.32, p<.002]$. Analysis of the interaction with the least significant difference $(L S D)$ test showed that contrast was larger in the animals with the added flavor $(p<.05)$.

There was also differential recovery from contrast [shift $\times$ flavor $\times$ day, $F(3,45)=5.41, p<.003$ ], which indicated that the contrast effect was reliable across all 4
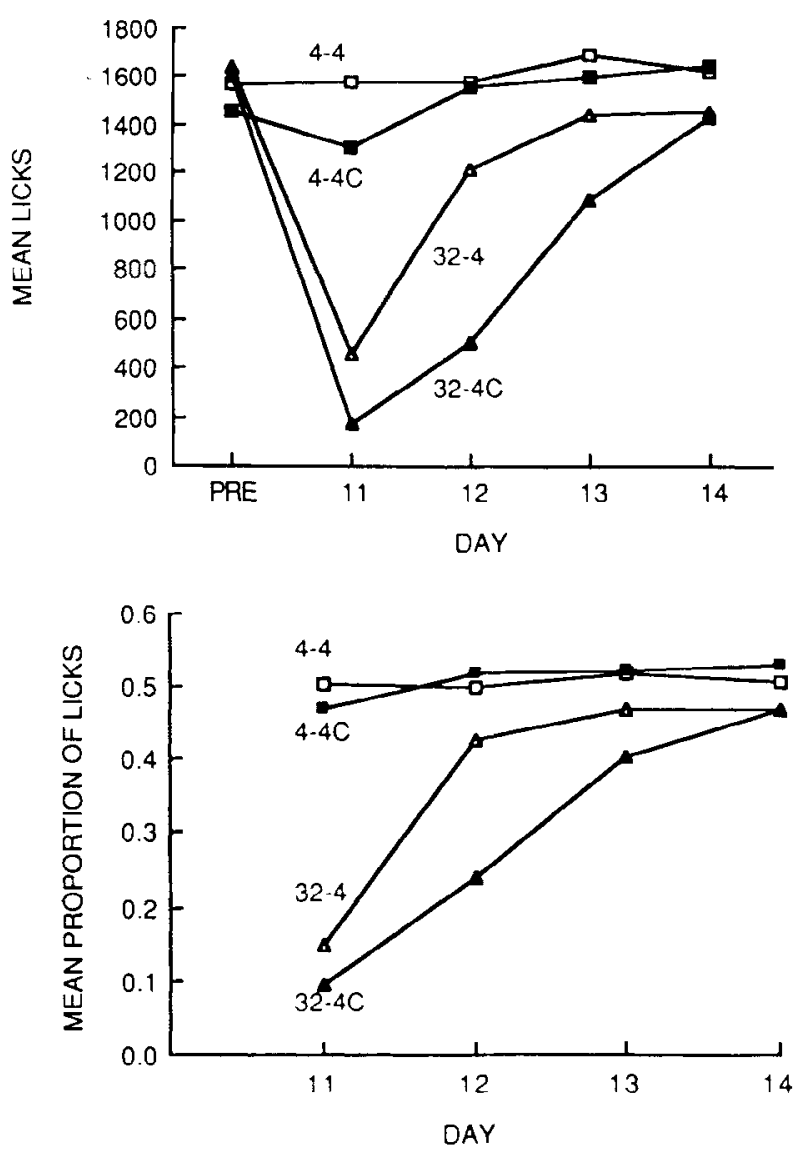

Figure 1. Top: Mean lick frequency at the end of the preshift period (PRE) and on each postshift day in Experiment 1 as a function of shift condition (shifted, 32-4, and unshifted, 4-4) and as a function of the presence or absence of a novel cherry flavor (C) in the postshift period. Bottom: Same data plotted in terms of proportion of preshift lick frequency [postshift/(postshift + terminal preshift)l.

postshift days in the flavor-added animals, but only on the first 2 postshift days in the nonflavor animals.

\section{Discussion}

The added flavor served to enhance contrast. The fact that the flavor differentially influenced the shifted animals supports the earlier finding that the experience of a contrast effect sensitizes shifted animals to novel flavors (Lombardi, 1980).

Since a previous experiment had shown that the introduction of a novel tone in the postshift period led to a reduced contrast effect (Lombardi \& Flaherty, 1978), it is clear that intrinsic (flavor) context changes produce quite different effects on contrast than do extrinsic (ambient) context changes. The next experiment involved a further investigation of extrinsic context change.

\section{EXPERIMENT 2}

In this experiment, the context was changed for half of the shifted and unshifted animals at the time of the shift. 
The change consisted of sprinkling wood chips beneath each test box, increasing the brightness of the lights in the test room, and adding white sheets of paper to the wall in front of the clear Plexiglas test apparatus.

\section{Method}

Subjects. Twenty-four male Sprague-Dawley-derived rats purchased from Blue Spruce were used as subjects. The animals were maintained as in the first experiment.

Apparatus. The apparatus was the same as that used in Experiment 1.

Procedure. The general procedure was the same as that used in Experiment 1, except that no taste manipulations were employed. The context manipulation, carried out during the postshift period period (Days 11-14), consisted of sprinkling wood chips beneath the test cages, increasing the illumination in the room, and adding white sheets of paper to the wall in front of the test chamber. These changes were made for half of the shifted and unshifted animals; the remaining animals continued under the same conditions that existed in the preshift stage.

\section{Results and Discussion}

Mean terminal preshift and daily postshift lick frequencies are presented in Figure 2. The shift in sucrose concentration led to an overall negative contrast effect $[F(1,20)=32.04, p<.001]$, which did not differ as a function of context change (shift $\times$ context, $F<1$ ). There was also no overall effect of context $(F<1)$. There was a reliable shift $\times$ context $\times$ day interaction $[F(3,60)=7.39, p<.001]$, which indicated that contrast was reliable over 3 postshift days in the contextchange group, but only over the first 2 days in the group shifted without a context change ( $L S D$ test, $p<.05$ ). The difference between the two groups of shifted animals on Postshift Day 1 (context change vs. no context change) was not reliable by $L S D$ test $(p<.05)$. Analysis of the data in terms of proportion of preshift lick frequency yielded similar results.

Thus, the context manipulation used in this experiment had little overall effect on contrast, except perhaps to prolong the duration of contrast (but this effect was small).

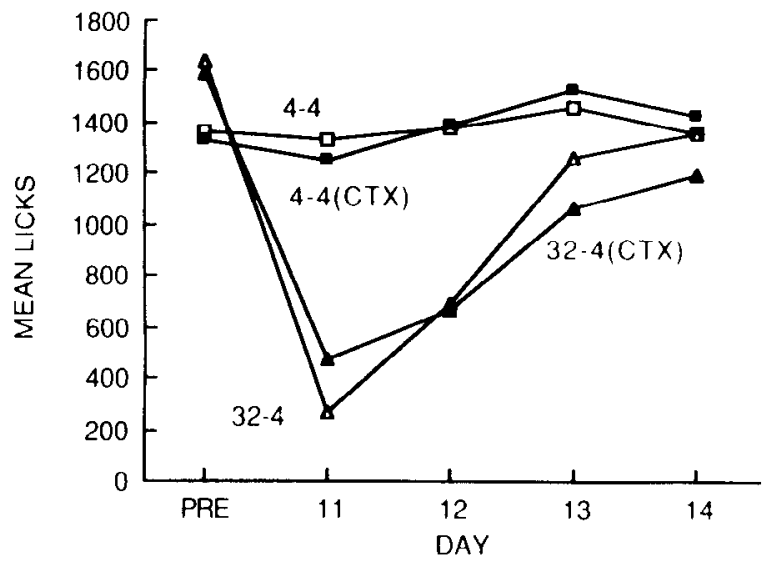

Figure 2. Terminal preshift (PRE) and daily postshift lick frequency in Experiment 2 as a function of shift condition (32-4 vs. 4-4) and the presence (CTX) or absence of a context change coincident with Postshift Day 1.

\section{EXPERIMENT 3}

This experiment was similar to Experiment 2, except that the context shift was made greater by moving half of the shifted and unshifted animals to a different room and different apparatus coincident with the reward shift.

\section{Method}

Subjects. Twenty-four male Sprague-Dawley-derived rats purchased from Blue Spruce were used as subjects. They were maintained as in the earlier experiments.

Apparatus. During the preshift phase of the experiment, the animals were tested in three identical clear Plexiglas chambers measuring $30 \times 25 \times 25 \mathrm{~cm}$. Two $1.5-\mathrm{cm}$ holes had been drilled in one side of each box, $21.7 \mathrm{~cm}$ apart and $4 \mathrm{~cm}$ above the wire-mesh floor. Lighting was provided by the ambient light in the room (approximately $538 \mathrm{~lx}$ ) and white noise permeated the room. The appropriate solution was delivered to the same side hole for all test days.

In the postshift phase of the experiment, half of the animals were tested in another room and in a different apparatus. This apparatus consisted of three identical Plexiglas chambers, $24 \mathrm{~cm}$ on each side and $20 \mathrm{~cm}$ high. These chambers were housed in acoustically insulated wooden boxes, with lighting provided by a houselight (GE 1829) and with white noise presented over a speaker contained within the chamber. Graduated drinking tubes were mounted so that the glass drinking spout was centered in a 1.4-cm hole drilled in one wall of the chamber. The orifice of the spout was $6.5 \mathrm{~cm}$ above the wire-mesh floor. This context-shift apparatus is referred to below as the "wooden box" apparatus. The animals" licking behavior was measured by a contact-relay circuit.

Procedure. The procedure, other than that involved with the context manipulation, was the same as that used in Experiment 2. Following the 10-day preshift period, half of the shifted and unshifted animals were continued in the same apparatus during the postshift period; the remaining animals were given the postshift solution in the different apparatus (wooden box).

\section{Results}

Mean terminal preshift and daily postshift lick frequencies are presented in Figure 3. The shift to a totally new context led to a substantial decline in consummatory behavior in both the shifted and the unshifted animals $[F(1,15)=9.90, p<.01]$. There was no overall contrast effect $[F(1,15)=1.67, p>.20]$, nor was there a shift $\times$ context interaction $(F<1)$. There was, however, a reliable shift $\times$ context $\times$ day interaction $[F(3,43)=$ $8.23, p<.001]$. Subsequent analysis of this interaction ( $L S D$ test, $p=.05$ ) showed that a reliable contrast effect occurred on Postshift Day 1 in the animals that did not have a context change, but the contrast was reliable on no other day in this group. In the case of the contextchange group, contrast was reliable on Postshift Day 2, but on no other day.

A similar pattern was obtained when the data were analyzed in terms of proportion of preshift lick frequency.

\section{Discussion}

The context change in this experiment, unlike that in Experiment 2, had a substantial effect on consummatory behavior. However, the fact that a contrast effect still occurred in the context-shift group, albeit not until Postshift Day 2, suggests that the memory of the preshift solution is not tied to the context in which that solution was ex- 


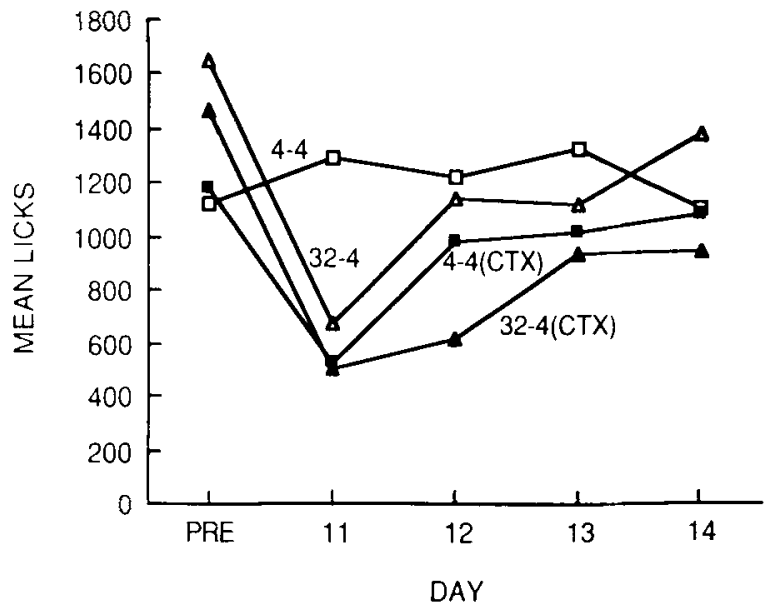

Figure 3. Terminal preshift (PRE) and daily postshift lick frequency in Experiment 3 as a function of shift condition (32-4 vs. 4-4) and the presence (CTX) or absence of a context change coincident with Postshift Day 1.

perienced. Although suggestive, the results of the present experiment are somewhat difficult to interpret because of the large decline in lick frequency produced by the context change itself. In the next experiment, an attempt to control this problem was made by giving the animals a period of habituation to the context-change apparatus prior to the start of preshift training.

\section{EXPERIMENT 4}

This experiment was similar to Experiment 3, except that all animals were habituated to the novel environment (wooden box) prior to the start of preshift training. This period of habituation was included to reduce the disruptive effects of the context change. It could be argued that such a context preexposure period should interfere with the conditioning of a context-sucrose memory but, in this experiment, all experience with the preshift solution was given in another context.

\section{Method}

Subjects. Twenty-four male Sprague-Dawley-derived rats purchased from Blue Spruce were used as subjects. They were maintained as in the earlier experiments.

Apparatus. The same two contexts used in Experiment 3 were used in this experiment.

Procedure. Except for the context manipulation, the procedure was similar to that of Experiment 3. In this experiment, all animals were given an initial 10-day period of habituation in the apparatus (wooden boxes) that would later function as the context-change condition for half of the animals. Following this initial period, all animals were given a 1-day habituation period to the acquisition apparatus. Following this, all animals had the 10 -day preshift treatment, half receiving $32 \%$ sucrose and half receiving $4 \%$ sucrose. In the postshift period, half of the animals were tested in the acquisition context and half were shifted to the context-change apparatus (wooden boxes). Postshift training was continued for 4 days.

\section{Results and Discussion}

The shift in sucrose concentration led to a reliable negative contrast effect in both the context-change and the noncontext-change animals $[F(1,20)=9.06, p<.01]$. This contrast effect did not vary as a function of the context change $(F<1)$. The context change itself produced a reliable and statistically equivalent decrement in licks in both the shifted and the unshifted animals $[F(1,20)=$ $13.30, p<.002]$. There was no differential rate of recovery from contrast as a function of context change (shift $x$ context $\times$ day, $F<1$ ). These data are illustrated in Figure 4.

Thus, the period of preshift habituation served to reduce the initial degree of disruption produced by the context shift (compared to that which occurred in Experiment 3), but there was still an enduring effect of the context change. However, degree of contrast did not vary as a function of context change, even though the context change itself clearly affected licking behavior.

Comparison of Figures 1-4 suggests that the long period of habituation used in the first phase of this experiment may itself have had an effect on contrast. That is, the decrement in lick frequency subsequent to the sucrose shift was apparently substantially less in Experiment 4 than in the three earlier experiments. Although this effect was not anticipated and the experiment was not designed to test for such an effect, it seemed worthwhile to conduct post hoc $t$ tests in order to obtain an estimate of the reliability of this apparent finding. Collapsed across contextchange conditions, the decrement in lick frequency subsequent to the shift was smaller in Experiment 4 than in Experiment $3[t(21)=2.63, p<.02]$, smaller than in Experiment $2[t(22)=4.73, p<.001]$, and smaller than the decrement obtained in the nonflavor group of Experiment $1[t(16)=2.62, p<.02]$. The degree of decrement was also smaller than that obtained in a population

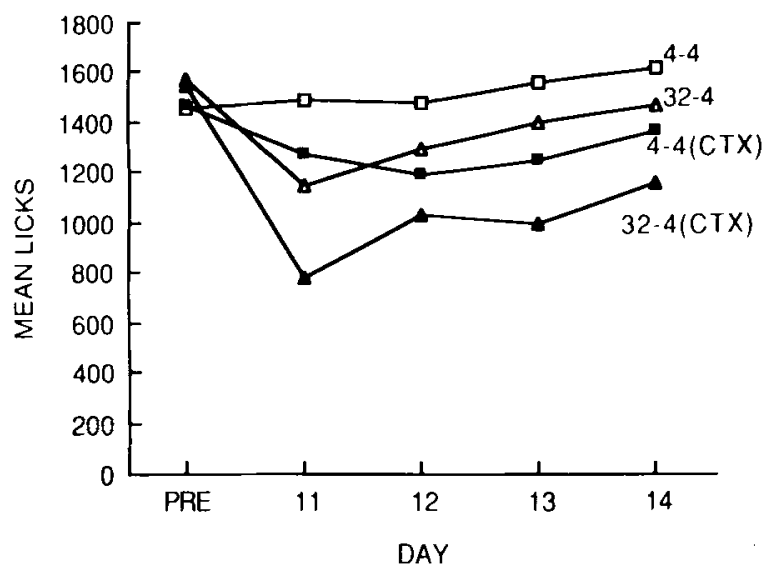

Figure 4. Terminal preshift (PRE) and daily postshift lick frequency in Experiment 4 as a function of shift condition (32-4 vs. 4-4) and the presence (CTX) or absence of a context change coincident with Postshift Day 1. 
of 397 Sprague-Dawley-derived rats run under notreatment conditions in our laboratory $[t(11)=3.60$, $p<.01]$.

This reduced shift may be related to the "initial nonreward" effect previously demonstrated in runway extinction studies. That is, preceding runway acquisition training with a series of nonrewarded placements in the runway retards subsequent extinction (Brooks, 1980; Capaldi \& Haggbloom, 1974; Spear, Hill, \& O'Sullivan, 1965; Spear \& Spitzner, 1967). In a particularly relevant study, Franchina and his colleagues demonstrated that the initial nonreward effect was not affected by differences between the apparatus used in the nonreward phase and that used in the acquisition and extinction phases under conditions in which the nonrewarded preexposure consisted of the animals being placed in the goalbox (Franchina, Weeks, \& Pais, 1977). The results of the present experiment suggest a similar outcome.

Since the series of experiments reported here was not designed to test the effects of habituation on contrast, and since this effect was not evaluated by the inclusion of nonhabituated controls within the same experiment, it is not appropriate to consider detailed theoretical interpretations of the initial nonreward effect and this apparent habituation effect. It remains as a serendipitous and potentially interesting by-product of the present experiments, one that requires further investigation.

\section{GENERAL DISCUSSION}

Experiments 2-4 suggest that a change in the ambient context, if it is of sufficient degree, will disrupt licking behavior but will not alter degree of contrast (cf. Capaldi, 1978). This finding suggests that the animals' expectancy of preshift reward quality is not necessarily associated with context cues. Thus, rats seem to be able to compare the value of a current reward with the memory of a preceding reward even if the two rewards are experienced in substantially different contexts.

It is certainly not the case that context cues are generally not associable with taste stimuli. For example, rats will learn to run at different speeds in a runway for different sucrose concentrations (e.g., see Collier \& Marx, 1959; Flaherty, Riley, \& Spear, 1973); bees demonstrate a variety of associative phenomena with exteroceptive stimuli paired with sucrose solutions (Bitterman, 1988; Couvillon \& Bitterman, 1984); contextual stimuli may modulate conditioned taste aversions (e.g., see Revusky \& Parker, 1976), although cues closely correlated with the taste substance, such as the bottle or nozzle, are apparently more potent than other apparatus-related stimuli (Archer, Sjoden, \& Nilsson, 1985); and, in our own situation, context-taste associations are indicated by differential latencies to initiate licking as a function of sucrose or saccharin concentration differences (e.g., Czachowski, Weaver, \& Flaherty, 1989; Flaherty \& Grigson, 1988). Thus, even though rats may readily form associations between taste stimuli and contextual cues, these associations are apparently not necessary for the comparison of reward value across different contexts.

This ability both to learn a signaling relationship between context and food value and to compare food value independent of context is consonant with behavior in foraging situations where bees, for example, compare nectar values across different flower patches (Pyke, 1978; Waddington \& Heinrich, 1981). The comparison of the energy value of food sources obtainable in different contexts is an implication of the marginal value theorem of optimal foraging theory (Charnov, 1976), and such a comparison has been demonstrated in rats as well as in bees. Mellgren, Misasi, and Brown (1984), for example, showed that rats would forage from a variety of different "patches" until each patch was no better in terms of food quantity than any other available patch.

The results obtained in Experiment 1, when taste context was varied, were different from those obtained in Experiments 2-4, when exteroceptive context was varied. In Experiment 1, the added flavor enhanced contrast by differentially reducing the lick frequency of the shifted animals. Since only the shifted animals were affected, it cannot be argued that the flavor simply lowered the reward value of the $4 \%$ sucrose solution. Rather, it seems that the experience of the reward shift sensitized the rats to other taste-related changes-an effect demonstrated previously when rats were shifted from $32 \%$ to $4 \%$ and then to $12 \%$ sucrose with an added novel flavor (Lombardi, 1980). Thus, experience with reward reduction, like experience with a toxin, seems to enhance the rat's natural tendency to neophobia (Barnett, 1975; Mitchell, 1976).

Since a change in neither the ambient context nor the taste context had a disinhibitory-like effect (reduced contrast), the results obtained in the earlier experiment (Lombardi \& Flaherty, 1978), which seemed to provide clear evidence of disinhibition, must be reconsidered. The evidence of disinhibition was produced by presenting a loud, pulsating, pure-tone stimulus within the animals' usual testing context. It is possible that the tone stimulus was more "intense" than the stimulus changes used in the present experiments. However, it does not seem feasible to scale the context changes used here on a dimension of intensity. In any case, Brimer (1970) reported ambiguous results when intensity was manipulated, finding that there was no substantial relationship between the intensity of a noise stimulus and disinhibition in one series of studies (p. 213), but also reporting a direct relationship between the degree of initial suppression produced by a stimulus and the subsequent disinhibitory effect produced by the same stimulus, a correlation that may have been partially based on an intensity dimension (pp. 216-217).

Another possible explanation of the difference in results obtained in the disinhibiting experiments and the present experiments is the relative novelty of the added stimulus. However, Brimer (1970) found that novelty was not an important parameter in his experiments and, in addition, it would seem that the added cherry flavor in Experiment 1 would have been as novel as the auditory stimu- 
lus used in the Lombardi and Flaherty (1978) experiments (a pilot study found that a 1:40 mixture of the cherry flavor with sucrose, rather than the 1:50 mixture used in Experiment 1 , reduced intake in both shifted and unshifted animals).

Perhaps the disinhibitory result obtained in the Lombardi and Flaherty (1978) experiments is related to a concatenation of events-an intense, punctate, nontaste stimulus presented in a familiar context. In any case, the present results demonstrate that context change itself is not disinhibiting in a negative contrast paradigm and that rats are able to compare rewards across substantially different contexts.

\section{REFERENCES}

Archer, T., Sjoden, P., \& Nilsson, L. (1985). Contextual control of taste-aversion conditioning and extinction. In P. D. Balsam \& A. Tomie (Eds.), Context and learning (pp. 225-272). Hillsdale, NJ: Erlbaum.

BARNETT, S. A. (1975). The rat: A study in behavior. Chicago: University of Chicago Press.

BitTerman, M. E. (1988). Vertebrate-invertebrate comparisons. In H. J. Jerison \& I. Jerison (Eds.), Intelligence and evolutionary biology (NATO ASI series, Vol. G17, pp. 251-278). Berlin: Springer-Verlag.

BoutoN, M. E. (1986). Differential control by context in the inflation and reinstatement paradigms. Joumal of Experimental Psychology: Animal Behavior Processes, 10, 56-74.

Bouton, M. E. (in press). Context and retrieval in extinction and other examples of interference in simple associative learning. In L. Dachowski \& C. F. Flaherty (Eds.), Current topics in animal leaming: Brain, emotion and cognition. Hillsdale, NJ: Erlbaum.

BRIMER, C. J. (1970). Disinhibition of an operant response. In R. A. Boakes \& M. S. Halliday (Eds.), Inhibition and learning (pp. 205227). London: Academic Press.

Brooks, C. I. (1980). Effect of prior nonreward on subsequent incentive growth during brief acquisition. Animal Learning \& Behavior, 8, 143-151.

CAPALDI, E. D. (1978). Effects of changing alley color on the successive negative contrast effect. Bulletin of the Psychonomic Society, 12, 69-70.

CaPaldi, E. J., \& HAGGBLOOM, S. J. (1974). Influence of reward magnitude on the initial nonreward effect. Bulletin of the Psychonomic Society, 3, 219-221.

Charnov, E. L. (1976). Optimal foraging: The marginal value theorem. Theoretical Population Biology, 9, 129-136.

Collier, G. H., \& MARX, M. H. (1959). Changes in performance as a function of shifts in the magnitude of reinforcement. Journal of Experimental Psychology, 57, 305-309.

Couvillon, P. A., Bitterman, M. E. (1984). The overlearning extinction effect and successive negative contrast in honeybees. Journal of Comparative Psychology, 98, 100-109.

Czachowski, C., Weaver, M., Flaherty, C. F. (1989, March). Effect of free access to ethanol on contrast effects in consummatory behavior. Paper presented at the meeting of the Southern Society for Philosophy and Psychology, New Orleans.

FlaherTy, C. F., \& AvDZEJ, A. (1976). Transsituational negative contrast. Animal Learning \& Behavior, 4, 49-52.

FlaherTy, C. F., BeCKER, H. C., \& CHECKE, S. (1983). Repeated suc- cessive contrast in consummatory behavior with repeated shifts in sucrose concentration. Animal Learning \& Behavior, 11, 407-414.

FLAHERTY, C. F., \& GRIGSON, P. S. (1988). From contrast to reinforcement: Role of response contingency in anticipatory contrast. Journal of Experimental Psychology: Animal Behavior Processes, 14, 165-176.

FlaherTy, C. F., \& Lombardi, B. R. (1977). Effect of prior differential taste experience on retention of taste quality. Bulletin of the Psychonomic Society, 9, 391-394.

Flaherty, C. F., Riley, E. P., \& Spear, N. E. (1973). Effect of sucrose concentration and goal units on runway behavior in the rat. Learning \& Motivation, 4, 163-175.

Franchina, J. J., Weeks, J. R., JR., \& Pais, N. (1977). Goalbox placements with initial nonreward and situational similarity in resistance to extinction. American Journal of Psychology, 90, 291-298.

Gonzalez, R. C., Fernhoff, D., \& David, F. G. (1973). Contrast, resistance to extinction, and forgetting in rats. Journal of Comparative \& Physiological Psychology, 84, 562-571.

Kaye, H., Preston, G. C., Szabo, L., Druiff, H., \& Mackintosh, N. J. (1987). Context specificity of conditioning and latent inhibition: Evidence for a dissociation of latent inhibition and associative interference. Quarterly Journal of Experimental Psychology, 39B, 127-145.

LOMBARDI, B. R. (1980). Enhanced neophobia induced by incentive contrast. Animal Learning \& Behavior, 8, 617-620.

LOMBARDI, B. R., FlaHERTY, C. F. (1978). Apparent disinhibition of successive but not of simultaneous negative contrast. Animal Learning \& Behavior, 6, 30-42.

Lovibond, P. F., Preston, G. C., \& Mackintosh, N. J. (1984). Context specificity of conditioning, extinction, and latent inhibition. Journal of Experimental Psychology: Animal Behavior Processes, 10, 360-375.

Meinrath, A. B., \& Flaherty, C. F. (1988). Effect of varied taste experience on negative contrast. American Journal of Psychology, 101, 87-96.

Mellgren, R. L., Misasi, L., \& Brown, S. W. (1984). Optimal foraging theory: Prey density and travel requirements in Rattus norvegicus. Journal of Comparative Psychology, 98, 142-153.

MitChELl, D. (1976). Experiments on neophobia in wild and laboratory rats: A reevaluation. Journal of Comparative \& Physiological Psychology, 90, 190-197.

Premack, D. (1969). On some boundary conditions of contrast. In J. T. Tapp (Ed.), Reinforcement and behavior (pp. 122-145). New York: Academic Press.

Pyke, G. H. (1978). Optimal foraging: Movement patterns of bumblebees between infloresences. Theoretical Population Biology, 13, 72-98.

Revusky, S., \& PARKer, L. A. (1976). Aversions to unflavored water and cup drinking produced by delayed sickness. Journal of Experimental Psychology: Animal Behavior Processes, 2, 343-353.

SPEAR, N. E. (1967). Retention of reinforcer magnitude. Psychological Review, 74, 216-234.

Spear, N. E., Hill, W. F., \& O'Sullivan, D. J. (1965). Acquisition and extinction after initial trials without reward. Joumal of Experimental Psychology, 69, 25-29.

SPEAR, N. E., \& SPITZNER, J. H. (1967). Effect of initial nonrewarded trials: Factors responsible for increased resistance to extinction. Joumal of Experimental Psychology, 74, 525-537.

Waddington, K. D., \& Heinrich, B. (1981). Patterns of movement and floral choice by foraging bees. In A. C. Kamil \& T. D. Sargent (Eds.), Foraging behavior: Ecological, ethological, and psychological approaches (pp. 215-230). New York: Garland.

(Manuscript received June 21, 1989; revision accepted for publication January 3, 1990.) 